Regular article

\title{
A gold nanoparticle piezoelectric immunosensor using a recombinant antigen for detecting Leishmania infantum antibodies in canine serum
}

\author{
Joilson Ramos-Jesus ${ }^{\mathrm{a}, \mathrm{b}}$, Lain Carlos Pontes-de-Carvalho ${ }^{\mathrm{c}}$, Stella M. Barrouin Melo ${ }^{\mathrm{d}}$, \\ Neuza M. Alcântara-Neves ${ }^{\mathrm{e}}$, Rosa F. Dutra ${ }^{\mathrm{a}, *}$ \\ a Biomedical Engineering Laboratory, Federal University of Pernambuco, Av. Prof. Moraes Rego, 1235 Recife, Pernambuco, Brazil \\ b Biodiversity and Biotechnology Research Group, Federal University of Piauí - UFPI, Parnaíba, Brazil \\ ' Gonçalo Moniz Research Center, Salvador, Bahia, Brazil \\ d Department of Veterinary, Federal University Bahia, Salvador, Brazil \\ e Department of Biointeraction, Federal University of Bahia, Salvador, Bahia, Brazil
}

\section{A R T I C L E I N F O}

\section{Article history:}

Received 22 July 2015

Received in revised form

27 December 2015

Accepted 29 January 2016

Available online 1 February 2016

\section{Keywords:}

Immunosensor

Quartz crystal microbalance

Leishmaniasis

Leishmania infantum

\begin{abstract}
A B S T R A C T
Visceral leishmaniasis $(\mathrm{VL})$ is a severe systemic and infectious disease potentially fatal when undiagnosed or untreated. So far, accurate diagnosis is still a problem, especially in endemic areas and in the tracking and screening of asymptomatic dogs, which are good reservoirs and main host in urban areas. Recombinant antigens based on DNA technology have provided more reliable serological diagnostics since the specificity can be achieved easier than using whole extracts. This work reports a sensitive piezeolectric immunosensor for anti-Leishamnia antibodies based on rLci2B recombinant antigens immobilized on quartz crystal electrode. The electrode surface was assembled by a nafion film recovered by gold nanoparticles (AuNPs) to promote a greater amount of rLci2B due to the increase of surface area and stability on bonds. The immunosensor distinguished the positive VL canine serum showing a good linearity $(r=0.989$; $p<<0.01)$ and a low relative error $(<5 \%)$ at 1:400 to 1:1600 serum dilutions. The system based on AuNP achieved better results regarding sensitivity and reproducibility than the cysteamine-based immunosensor without AuNP $(r=0.769 ; p=0.147)$. The results obtained show as a promising tool for diagnosis of the VL.
\end{abstract}

(c) 2016 Elsevier B.V. All rights reserved.

\section{Introduction}

Visceral leishmaniasis (VL) or kala-azar is a chronic, debilitating and occasionally fatal disease that has an estimated incidence of 500,000 cases per year. This zoonosis is widely distributed endemically in 65 countries [1] and caused by a protozoan of the genus Leishmania, with an anthroponotic species (Leishmania donovani), occurring in Asia and Africa, and a zoonotic species (Leishmania infantum) occurring in the Mediterranean Region and South America, where it was previously called Leishmania chagasi (=syn. $L$. infantum). American visceral leishmaniasis VLA is considered solely caused by L. infantum [1], and transmitted by Lutzomyia longipalpis, a phlebotomid fly. The dogs have all the characteristics of a good reservoir for the parasite: they are present in the domestic and peridomestic environment working as a powerful source for the vector, and they develop high parasitic skin, allowing a high rate

\footnotetext{
* Corresponding author. Fax: +55 8121268200.

E-mail address: rosa.dutra@ufpe.br (R.F. Dutra).
}

of infection [2]. These characteristics are important to maintain the domestic cycle vector-dog-vector-human [3], The diagnosis of dogs with visceral leishmaniasis is critical to public health [4], and its epidemiological origin and symptoms should be considered [5].

So far, effective diagnosis of VL remains a challenge for the scientific community. Parasitological diagnosis based on visualization of the parasite is usually considered as the gold standard for disease diagnosis. However, this technique requires highly skilled personnel and the information is limited, particularly when parasitaemia is low. The polymerase chain reaction (PCR) test has proven to be an effective test for diagnosis of leishmaniasis [6-9], but also requires expensive reagents and equipment as well as skilled personnel, being not appropriate for fieldwork. Serological methods such as enzyme-linked immunosorbent assay (ELISA) sometimes combined to the immunofluorescence assay test (IFAT) to increase specificity has been usually employed [10-14], nevertheless a low reproducibility and specificity values are obtained due to the use of whole parasite extracts as antigens. Recently, Souza et al. (2012) [5] developed a ELISA serological test based on a purified recombinant Leishmania antigens (rLci2B) that confirmed 
a high sensitivity (96\%) and specificity (95\%) evaluating in a leishmaniasis canine serum panel $(n=256)$. The purified proteins did not present cross-reactivity with sera from dogs infected with Trypanosoma caninum, Babesia canis and Ehrlichia canis. Cross-reaction was verified only agaisnt sera from dogs infected with Leishmania brasiliensis (11,7\% for rLci2B) [5]. Based on ELISA results, use of rLci2B as antigens for diagnostic of canine leishmaniasis was recommended. Although the ELISA test has been widely diffused it does not considered a practical and economical method, beside to be a time-consuming and non-practical especially for screening and epidemiological studies.

On last few decades, a number of researchers have been devoted to the development of biological sensors for microorganism detections [15]. Such sensors offer several advantages because they not require pretreatment of the sample, have a low cost, ease of use, rapid detection and label-free or radiation-free reagents [16]. The quartz crystal microbalance (QCM) is a sensitive label-free and on-line detection of adsorbed analytes based on the linear relationship between the decreased resonant frequency of a piezoelectric quartz crystal and the mass deposited on its surface [17] QCM immunosensor has been widely used for investigations of biomolecular interactions and innumerous immunoassays [18-20]. However, extensive efforts are still necessary to improve the sensitivity and specificity of QCM-based immunosensors [21,22].

Nanomaterials have emerged as potential tools for biosensor development by the increase of the sensitivity of assays [23]. Gold nanoparticles (AuNPs), owning to the non-toxic nature and excellent biological compatibility, have recently attracted significant attention for a variety of biomedical applications [24,25]. AuNP enhances the sensitivity of sensors by increasing effective area of the electrode surface and promote a larger amount of immobilized antibody or antigen molecules, thus increasing the sensibility of the assay [16,26]. Additionally, AuNP is also a useful interface for the electrocatalysis of redox processes by increasing the electron transfer between redox proteins and bulk electrode material $[27,28]$.

In order to improve the binding capacity of bioreceptor and permit a stable platform, AuNPs are often conjugated to other materials like polymers and other compounds [29,30]. Nafion is a sulfonated tetrafluorethylene copolymer that acts as an ion exchanger with a preference for hydrophobic cations [31,32]. Besides, nafion is chemically inert and hydrophilic [31], showing ideal properties for the preparation of biosensors. Herein, nafion was used to anchor the cysteamine modified AuNP by strong linkage due to its sulfona group. Acting as a matrix support, nafion offers the advantage in simplicity and stability for AuNP attachment and it is an easy protein immobilization procedure. Aiming at the detection of infected dogs by L. infantum a label free immunosensor consisting of AuNP-nafion film and a selective recombinant antigen to provide an immunoassay with high sensitivity and specificity values is described.

\section{Materials and methods}

\subsection{Reagents}

Cysteamine (hydrochloride), glycine, bovine serum albumin (BSA) were supplied by Sigma-Aldrich (St Louis, MA, USA). Potassium hexacyanoferrate (III) was acquired from VETEC Inc. (São Paulo, SP, Brazil). Gold nanoparticles (AuNP) of approximately 3-5 nm were acquired from Sigma Aldrich (St. Louis, MA, USA). Nafion (polyanionic perfluorosulfonate) was acquired from Ion Power, Inc. (New Castle, DE, USA). All other reagents were of analytical grade. The water used to prepare all solutions was obtained from a Milli-Q water purification system (Millipore, Billerica, MA, USA).

\section{2. rLci2B antigen}

The $L$. chagasi/L. infantum recombinant antigen (rLci2B) was obtained and characterized as described by Ramos-Jesus et al. [20]. The recombinant protein was produced using the Escherichia coli. The process was registered in the National Institute of Industrial Property of Brazil (INPI) with number PI0900961-2. The rLci2B is a 293 amine acid long polypeptide with an $\mathrm{N}$-terminal six histidine tag having homology with parasite cytoskeleton protein kinesin. The recombinant antigen, showed with potential for serodiagnosis of $L$. chagasi/L. infantum according to ELISA, has been tested against sera from dogs with the following conditions: (a) 46 dogs naturally infected with Leishmania, detected by splenic aspirate and culture from endemic areas which were either polysymptomatic (21 dogs), oligosymptomatic (21 dogs) or asymptomatic (4 dogs); (b) 31 dogs from Leishmania-free areas: 7 with demodicosis; 4 with babesiosis; and 20 dogs with ehrlichiosis. All diseases were parasitologically confirmed. The infectious agent of all diseases was specifically confirmed, and assay for VL demonstrated sensitivity and specificity above $90 \%$ [33]. L. infantum kinesin was also tested by an ELISA kit showing as non-reactive to other species of Trypanosomatidae $[34,35]$.

\subsection{Canine serum samples}

The canine serum samples were obtained from dogs naturally infected with L. infantum from an endemic area, detected by spleen aspirate cultures. All infections were parasitological confirmed by ELISA assays. Sera from healthy mongrel dogs housed in a nonendemic area were used as controls (negative LV). The protocol for animal handling was approved by the Ethics Committee on Animal Use of the Gonçalo Muniz Research Center, Fundação Oswaldo Cruz, Bahia, Brazil.

\subsection{Equipments and measurements}

The experimental setup consisted of the Research Quartz Crystal Microbalance System - RQCM (Maxtec Inc.,USA) with a circuit that incorporate an adjustable crystal capacitance cancelation to reduce error caused by parasitic capacitance of the crystal. The RQCM was connected to a PGstat 1.2 potentiostat/galvanostat Autolab (Eco Chemie, Utrecht, Holland), both coupled to a microcomputer, which is controlled by the GPES program, version 4.9 (Eco Chemie, Utrecht, Holland).

The cyclic voltammograms were registered using a three electrode system, which was immersed in an electrochemical cell with $40 \mathrm{~mL}$ volume. The quartz crystal electrode $(9 \mathrm{MHz}$, Maxtec Inc., USA), as a working electrode, $\mathrm{Ag} / \mathrm{AgCl}$, as reference electrode and helicoidal platinum wire, as auxiliary electrode, were used. The electrochemical cell ( $40 \mathrm{~mL}$ volume) was filled by a redox probe solution of $5 \mathrm{mmol} \mathrm{L}^{-1} \mathrm{~K}_{3}\left[\mathrm{Fe}(\mathrm{CN})_{6}\right] / \mathrm{K}_{4}\left[\mathrm{Fe}(\mathrm{CN})_{6}\right]$ prepared in $0.1 \mathrm{mmol} \mathrm{L}^{-1}$ of $\mathrm{KCl}$, and the cyclic voltammetries were carried out at $100 \mathrm{mVs}^{-1}$ scan rate.

Change frequencies from the quartz electrode by adsorption were acquired at $1 \mathrm{~Hz}$ sampling rate. The quartz crystal electrode was setup in the EQCM chamber ( $400 \mu \mathrm{L}$ volume) coupled to two peristaltic pumps. Phosphate buffered saline (PBS, $50 \mathrm{mM}, \mathrm{pH} 7.2$ ) was used as carrier buffer and the temperature was $23^{\circ} \mathrm{C}$. For all measurements, the flow ( $300 \mathrm{uL} \mathrm{min}^{-1}$ ) was stopped and baseline stabilized when the coefficient of variation was less than $5 \%$. 
(I) Surface successively cleaned by $0.5 \mathrm{M} \mathrm{NaOH}$, ethanol and water
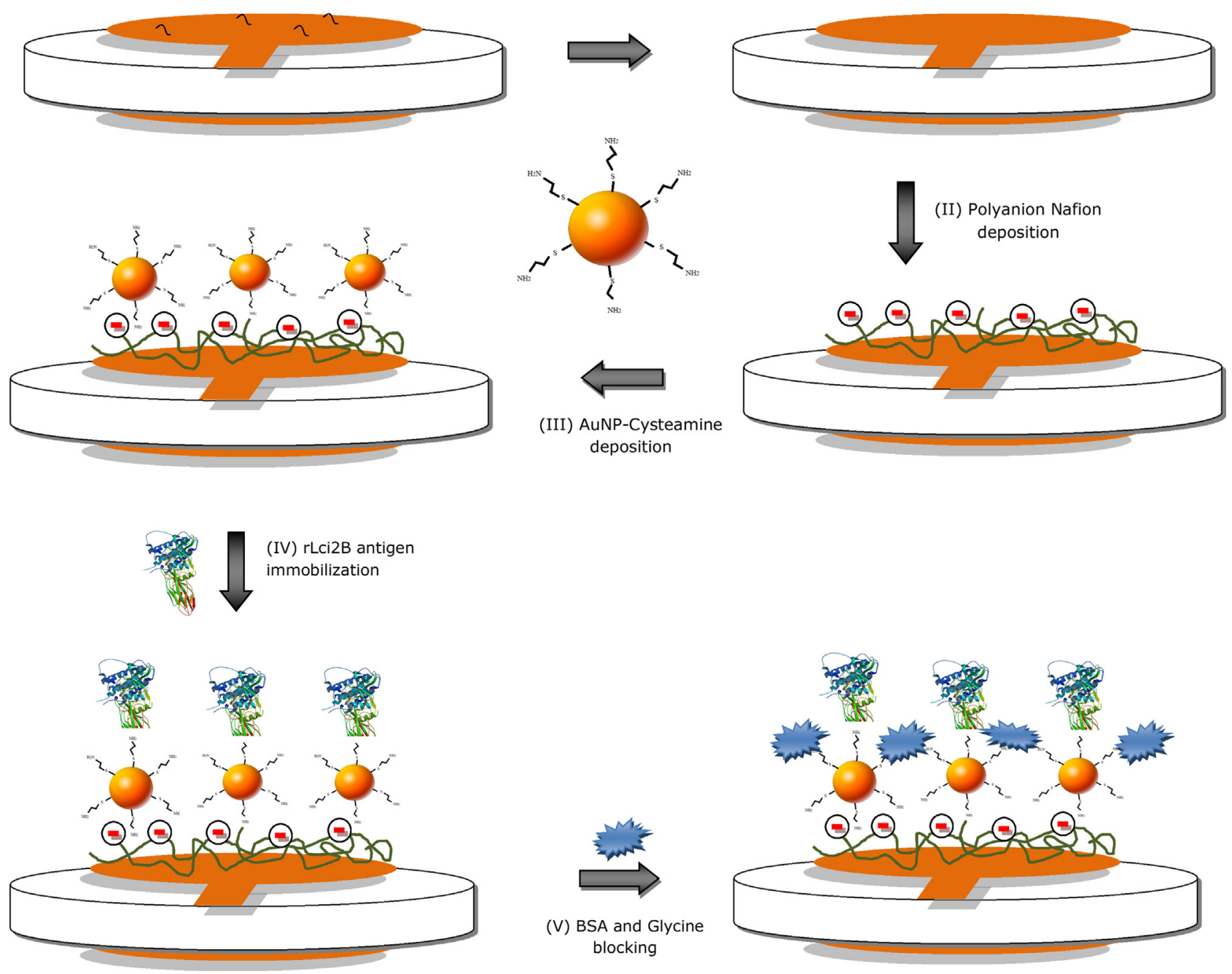

Fig. 1. Schematic representation of stepwise modification on the quartz crystal electrode for and rLci2B antigen immobilization.

\subsection{Preparation of the quartz crystal immunoelectrode}

Prior to modifications, the quartz crystal electrode was sequentially cleaned in $0.5 \mathrm{M} \mathrm{NaOH}$, ethanol and distilled water. Nafion film was obtained by the dip-coating method, in which the coated quartz crystal electrode was dipped in the solution of $5 \%(\mathrm{v} / \mathrm{v})$ nafion in ethanol, and was dried at room temperature for $2 \mathrm{~h}$, maintained in an excicator. Afterwards, a suspension containing the cysteamine modified AuNP was incubated for $1 \mathrm{~h}$ on the modified electrode. The amine-AuNP $[5.47 \times 109 / \mathrm{ml}]$ was achieved by transferring to a microtube $180 \mu \mathrm{l}$ of cysteamine solution ( $50 \mathrm{mMmmol} / \mathrm{l}$ ), prepared in $10 \mathrm{mM}$ phosphate buffered saline (PBS) $\mathrm{pH} 7.2$, and incubating for approximately $2 \mathrm{~h}$ at room temperature with $20 \mu$ l of AuNP solution [1:1] containing $2.7 \times 10^{12}$ per ml. To immobilize the antigen on the surface of the AuNP, the rLci2B was pre-incubated for one hour with a solution of 1-ethyl-3-[3-dimethylaminopropyl] carbodiimide hydrochloride (EDC) and $\mathrm{N}$-hydroxysulfosuccinimide (NHS). EDC reacts with carboxyl groups in the antigen, forming an intermediate aminereactive compound ( 0 -acylisourea). Afterwards, the modified gold electrode was incubated with rLci2B antigen $(3 \mu \mathrm{g} / \mathrm{mL})$ for $1 \mathrm{~h}$. After immobilization of the recombinant antigen, a solution [1:1] containing $50 \mathrm{mM} \mathrm{mmol} / 1$ glycine and $2.5 \% 25 \mathrm{~g} / \mathrm{l}$ BSA prepared in PBS was pipetted on the AuNP modified electrode and left to react for $45 \mathrm{~min}$. Schematic design of modifications on the electrode surface of immunosensor is shown in Fig. 1.

\subsection{Response of the immunosensor}

The analytical response of the immunosensor was evaluated by applying canine serum samples 1:400 diluted in PBS (pH 7.2) in the sampler. The samples were incubated on the active area of the quartz crystal electrode in stopped flow for $15 \mathrm{~min}$. Prior to the all measurement of frequencies, the quartz crystal electrode was washed with PBS for 5 min. Control tests with normal (negative) samples were performed as described. All measurements were conducted at room temperature.

\section{Results and discussion}

\subsection{Preparation of the quartz crystal immunoelectrode}

Nano-scale surfaces of AuNPs functionalized by thiol have recently been used for obtaining enhanced sensitivity of immunosensor. due to the well-defined self-assembly of thiolate at the surface of gold through Au-thiol binding that led to an 


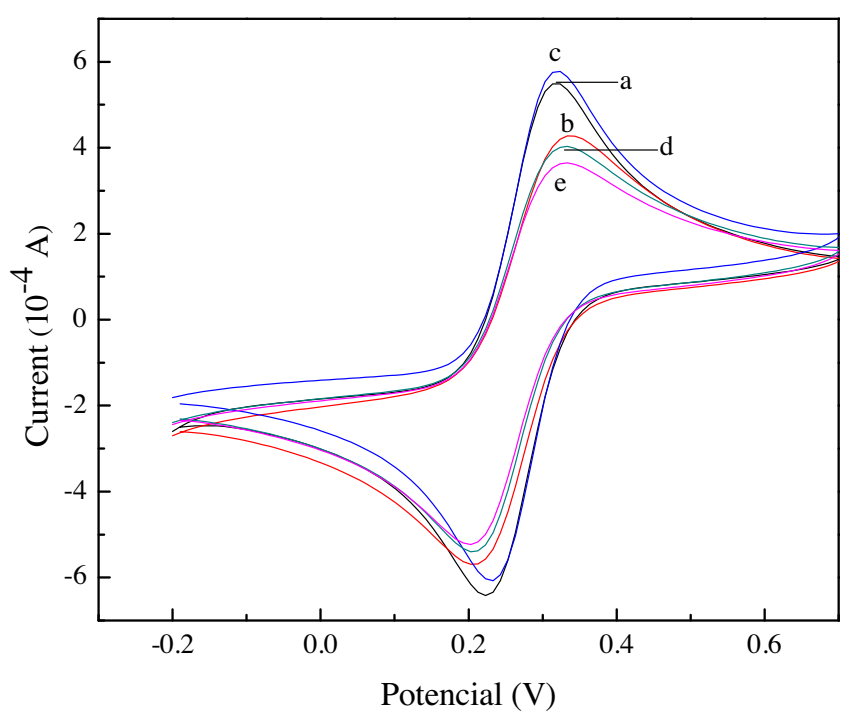

Fig. 2. Cyclic voltammogram of (a) bare electrode; (b) nafion film; (c) cysteaminegold nanoparticles; (d) rLci2B; (e) glycine and solution. Readings taken at pH 7.4; scanning speed: $100 \mathrm{mVs}^{-1}$.

increased effective surface to provide a sufficient amount of sites for binding[36]. In the present study, cysteamine was used to electrostatically bind AuNP to a nafion film on the electrode surface [37] and to provide a functionalized surface for immobilization of the recombinant antigen $[38,39]$. Thiols spontaneously adsorb on noble metals, particularly gold $(\mathrm{Au})$, in order to form an oriented structure [40,41]. On the other hand, amine groups in cysteamine provide ideal anchoring for covalent attachment of proteins. Preincubation of the antigen with the EDC solution allows EDC to react with carboxyl groups in the antigen to form an intermediate aminereactive $O$-acylisourea that is susceptible to hydrolysis, unstable and short-lived in aqueous solutions. NHS stabilizes the intermediate amine-reactive compound, converting it into an amine-reactive NHS ester [42,43], thereby increasing the efficiency of EDC coupling. This amine-reactive NHS ester reacts with the amine group of cisteamine present on the modified AuNP in a stable and organized manner, producing a conjugate of the two molecules joined by a stable amide bond. The response of the immunosensor is achieved by immobilized rLci2B antigen, which interacts with the anti- $L$. infantum antibodies present in canine sera.

The stepwise modification of the quartz crystal electrode was accomplished by cyclic voltammetry. The three electrodes were immersed in a beaker filled up with a $\left.0,1 \mathrm{mM} \mathrm{Fe}(\mathrm{CN})_{6}\right]^{3-/ 4-}$ solution, which acted as redox probe. Initially, after cleaning the electrode, well-defined characteristic waves of $\mathrm{Fe}(\mathrm{CN})_{6}{ }^{3-/ 4-}$ with peak-to-peak separation ( $\triangle E \mathrm{p}$ ) of about $0.099 \mathrm{~V}$ are seen on a bare Au electrode. There was a reduction in the area of cyclic voltammetry as a result of mass deposition and the blocking effect of the electron transfer of $\mathrm{Fe}(\mathrm{CN})_{6}{ }^{3-/ 4-}$ on the surface of the crystal during the formation of the nafion film on the crystal surface, antigen immobilization and the blockage of the nonspecific bindings. The increase in the current of the redox peaks confirms the presence of AuNP. The rLci2B immobilization was confirmed by decreasing on the redox peaks performed with a $\left[\mathrm{Fe}(\mathrm{CN})_{6}\right]^{3-/ 4-}$ as redox probe (Fig. 2).

Quantitative analysis of recovering can be calculated by cyclic voltammetric technique using as a redox probe an ionic solution of $\mathrm{Fe}(\mathrm{CN})_{6}{ }^{3-14-}$ [44-46]. The location and intensity of the redox peaks can be used in the characterization of surface modification with respect to the degree of coverage and stability. Fig. 2 displays successive cyclic voltammograms performed using $1 \mathrm{mMmmol} / \mathrm{l}$

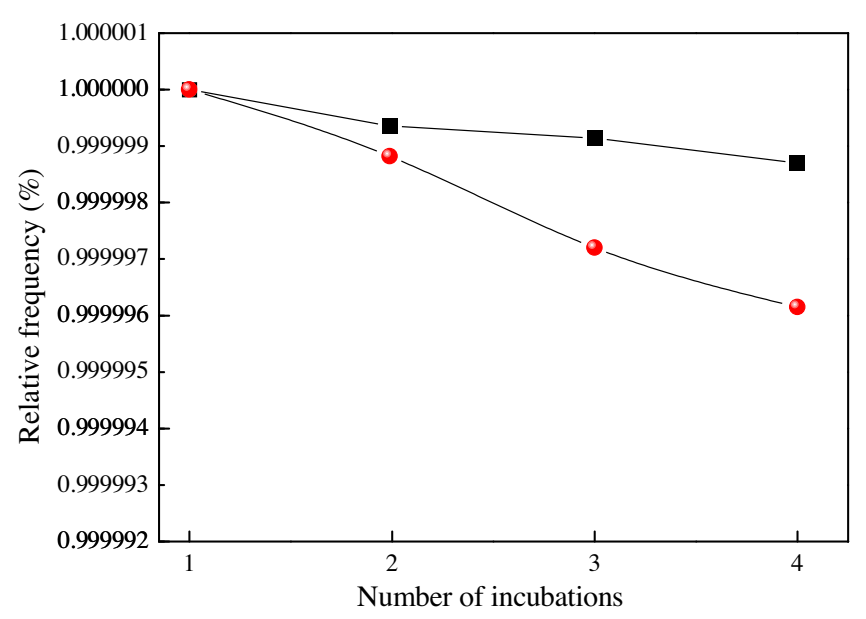

Fig. 3. Influence of AuNP on response of piezoelectric immunosensor at successive incubations of positive serum sample (1:400 dilution in PBS serum) for antiLeishmania infantum antibodies; (•) with $\operatorname{AuNP}(1: 10)$; (ロ) without AuNP.

of $\mathrm{K}_{3} \mathrm{Fe}(\mathrm{CN})_{6}{ }^{3 / 4-}$ in $0.1 \mathrm{M} \mathrm{mol} / 1$ of aqueous $\mathrm{KCl}$ as the redox probe. When the electrode surface is modified, the electron transfer kinetics of $\mathrm{Fe}(\mathrm{CN})_{6}{ }^{3-/ 4-}$ is perturbed. Compared with the response on a bare gold electrode, a reversibility and increase on the redox peaks were exhibited by the AuNP-cysteamine-modified nafion gold electrode due to specially to the attractive electrostatic forces between the protonated amine groups of cysteamine-AuNPs and nafion film [47]. These profiles are consistent with the enhanced electron transfer barriers introduced upon the increase of electroactive areas [48]. Besides, the AuNPs have a non-toxic nature and an excellent biological compatibility. The immobilization of rLci2B and blockage of non-specific binding on the gold qage uartz crystal electrode were confirmed by the decreasing of redox peaks [49]. The quantitative analysis of coverage can be estimated by cyclic voltammetry. Surface coverage $(\theta)$ is calculated by Eq. (1),

$\theta=1-\left(Q_{\text {Bare }} / Q_{E M}\right)$

where $Q_{\mathrm{Bare}}$ is the charge of the bare quartz crystal electrode and $Q_{E M}$ is the charge of the electrode modified by nafion, nanogoldcysteamine, blocking solution and the rLci2B antigen, calculated from the area under the peak redox of the cyclic voltammogram exhibited on Fig. 2. According to Eq. (1), the surface coverage of the electrode by nafion, the rLci2B antigen and blocking solution provided successively a decrease of redox by hindering the diffusion barrier of $28.26 \%, 36.14 \%, 51.26 \%$, respectively, compared to the bare electrode. Contrary, the AuNP-cysteamine layer promoted an increase of $4.32 \%$ compared to bare electrode. The presence of cysteamine on the surface of the AuNP allowed the rLci2B antigen to have an and retain its bioactivity. The findings revealed that the employment of AuNP expanded the effective area of the electrode, thereby improving the sensitivity of the assay. The amplification of the detection process is achieved through the interaction of the sensing surface previously covered by the nafion film with the AuNP-cysteamine conjugate, increasing the amount of amine groups on the electrode surface, which act as reactive sites for the rLci2B antigen. Thus, the frequency response associated with the antigen-antibody binding event is amplified by mass adsorption due to immunoreactions (Fig. 3).

\subsection{Optimization of experimental conditions}

The amount of AuNPs attached to the electrode surface and their capacity to immobilize antigens is strongly associated with the sensitivity [50]. Herein, the AuNPs were diluted in different ratios in 
Table 1

Immunosensor response according to concentration of the AuNP.

\begin{tabular}{lllll}
\hline AuNP/mL & $R^{2}$ & Intercept value/SD & Slope value/SD & $p$ \\
\hline $1: 2(2735 \mathrm{E} 12)$ & 0.979 & $9.031 \mathrm{E} 6 / 1.257$ & $-3.931 / 0.465$ & $\mathbf{0 . 0 1 4}$ \\
$1: 10(0.547 \mathrm{E} 12)$ & 0.994 & $9.064 \mathrm{E} 6 / 2.385$ & $-13.330 / 0.653$ & $\mathbf{3 . 4 E - 5}$ \\
$1: 20(0.2735 \mathrm{E} 12)$ & 0.989 & $9.009 \mathrm{E} 6 / 2.479$ & $-10.797 / 0.721$ & $\mathbf{1 . 2 E}-\mathbf{4}$ \\
Control (without AuNP) & 0.769 & $9.001 \mathrm{E} 6 / 11.514$ & $-7.874 / 3.409$ & $\mathbf{0 . 1 4 7}$ \\
\hline
\end{tabular}
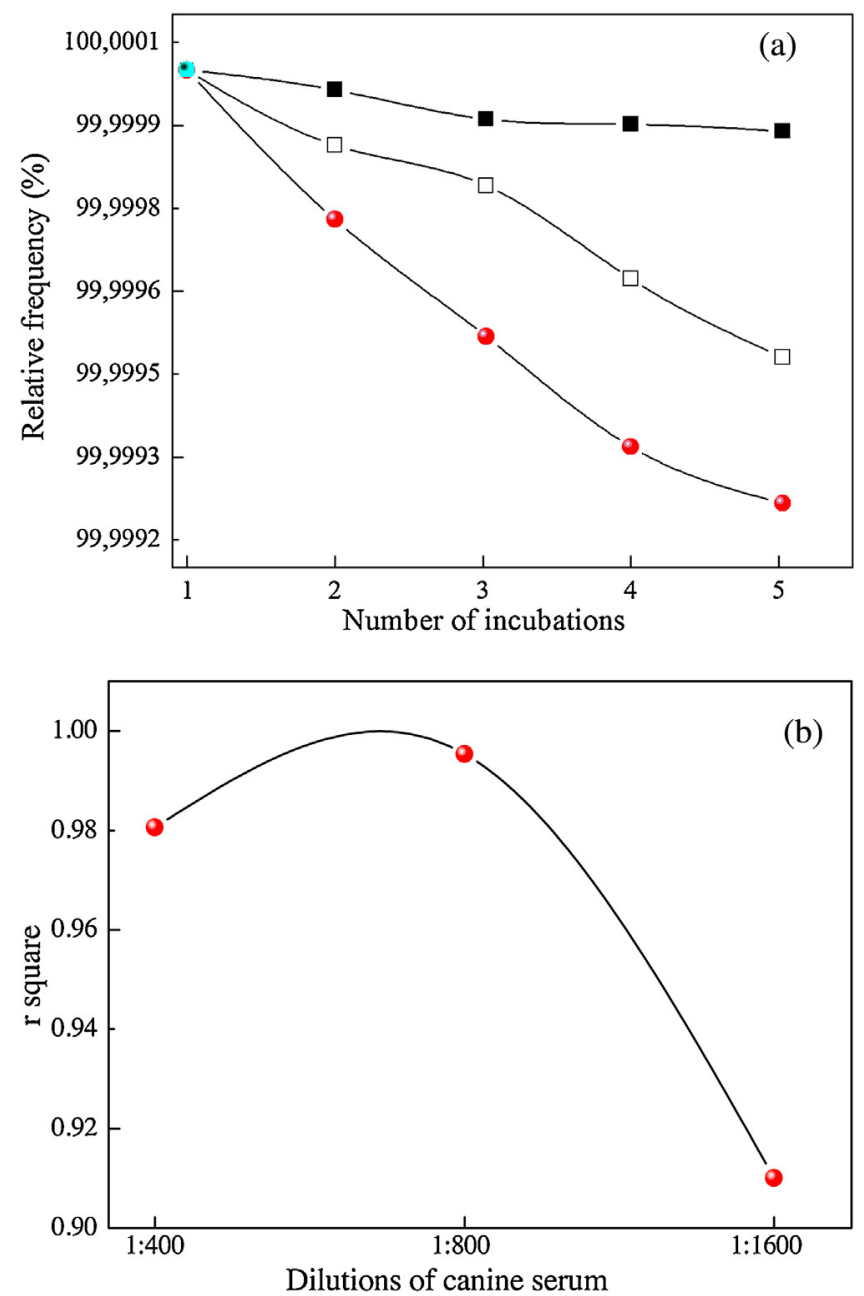

Fig.4. Frequency changes of immunosensor by successive incubations with positive serum samples at different PBS dilution $(\bullet) 1 / 400$ ( $\square$ ) 1/800 and (घ) 1/1,600 (a); and $r$ square plotted as function of serum dilutions (b).

order to determine the maximal sensitivity. The best result was obtained using ratios of 1:10 $(0.547 \times 10 \mathrm{E} 12)$ of AuNP (Table 1$)$. Analytical performance of immunosensor was established according to the potential to discriminate the positive serum. As shown in Fig. 4a, the immunosensor was capable to discriminate the samples diluted in PBS up to 1/800. At 1/1,600 PBS serum dilution, frequency changes of negative serum samples (control) were practically similar for all the incubations, considering the standard deviations. It means that the developed immunosensor presented an optimal performance at 1:400 serum dilutions. This statement is also confirmed by analysis of the coefficient of discrimination ( $r$ squared) obtained from the calibration curves at different dilution ratios. In dilutions up to $1: 800$, the $r$ square is significantly smaller than one unity indicating that the specificity is reduced and the immunosensor was not able to discriminate a positive canine $L$. chagasi serum (Fig. 4b).

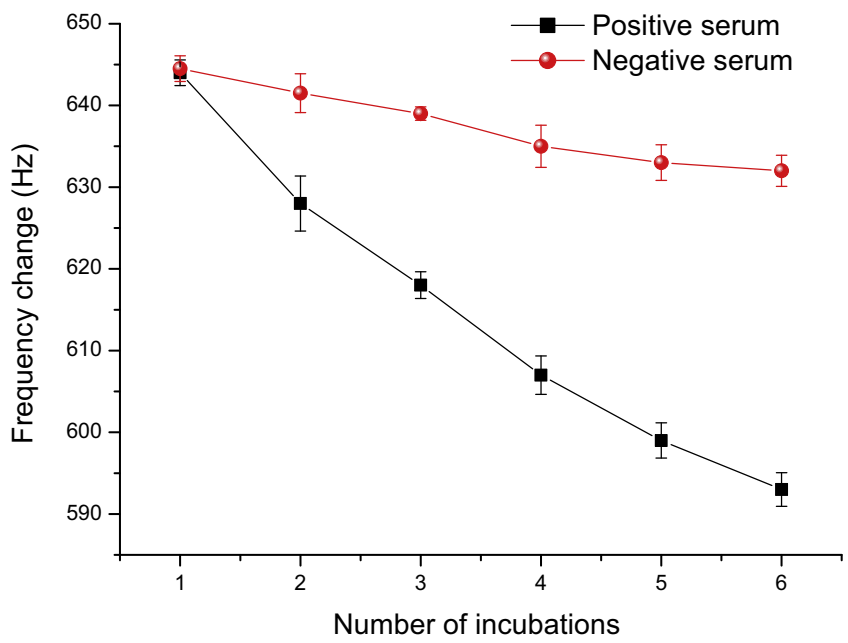

Fig. 5. Analytical curve of response to antibodies present in positive ( ) and negative (ם) canine serum for anti-Leishmania infantum antibodies under optimal experimental conditions.

Table 2

Statistical analysis of immunosensor response according to different solutions used to block nonspecific reactions.

\begin{tabular}{llllll}
\hline Blocking agent & Serum & $r$ & Slope value/SE & $p$ & Ef.b (\%) \\
\hline Glycine & + & 0.966 & $-9.7 \mathrm{E}-7 / 8.7 \mathrm{E}-8$ & $\mathbf{0 . 0 0 5}$ & 40.90 \\
& - & 0.908 & $-7.3 \mathrm{E}-7 / 2.2 \mathrm{E}-7$ & 0.191 & \\
BSA & + & 0.985 & $-1.9 \mathrm{E}-6 / 1.7 \mathrm{E}-7$ & $\mathbf{0 . 0 0 1}$ & 52.27 \\
\multirow{2}{*}{ Glycine + BSA } & - & 0.909 & $-8.1 \mathrm{E}-7 / 2.4 \mathrm{E}-7$ & 0.190 & \\
& + & 0.986 & $-1.0 \mathrm{E}-6 / 8 . \mathrm{E}-8$ & $\mathbf{0 . 0 0 1}$ & $\mathbf{9 5 . 5 3}$ \\
& - & -1.180 & $8.9 \mathrm{E}-9 / 3.2 \mathrm{E}-8$ & 0.196 & \\
\hline
\end{tabular}

The sensitivity of the immunosensor was measured through successive incubations of canine serum samples positive for $L$. infantum antibodies diluted at 1:400 in PBS. The positive serum samples were previously confirmed by absorbance measurements $(O D>2000)$ in indirect ELISA, according to Ramos-Jesus et al. The analytical curve showed a good linearity $(r=0.9889 ; p<<0.01)$, with a low relative error (5\%) (Fig. 5).

The selectivity of the immunosensor was tested by incubating positive and negative canine serum samples for anti- $L$. infantum antibodies diluted at 1:400 in PBS. A decreased frequency response was found when the immunosensor was incubated with canine serum positive for anti- L. infantum antibodies and a practically constant response was found when incubated with negative serum, demonstrating that the immunosensor is selective regarding the identification of anti-L. infantum antibodies in canine serum samples (Fig. 5). A number of authors have reported the role of blocking nonspecific bindings by some molecules [51,52]. In the present study, the use of different blocking agents resulted in different degrees of immunosensor selectivity. Fig. 6 summarizes the experimentally observed saturation levels of nonspecific binding obtained after exposure of the blocked surfaces to the L. infantum positive and negative canine serum samples using one of the three blocking solutions (PBS containing $50 \mathrm{mM}$ of glycine, PBS containing $2.5 \%$ BSA or PBS containing glycine plus BSA).

Table 2 summarizes a data comparative of the immunosensor responses to different blocking agents against nonspecific bindings. Blocking efficiency was calculated by Eq. (2), in which Ef.b is the blocking efficiency, $\triangle F s p$ is the delta frequency of positive serum and $\triangle F s n$ is the delta frequency of negative serum.

$E f . b(\%)=\frac{\Delta F s p-\Delta F s n}{\Delta F s p} \times 100$ 

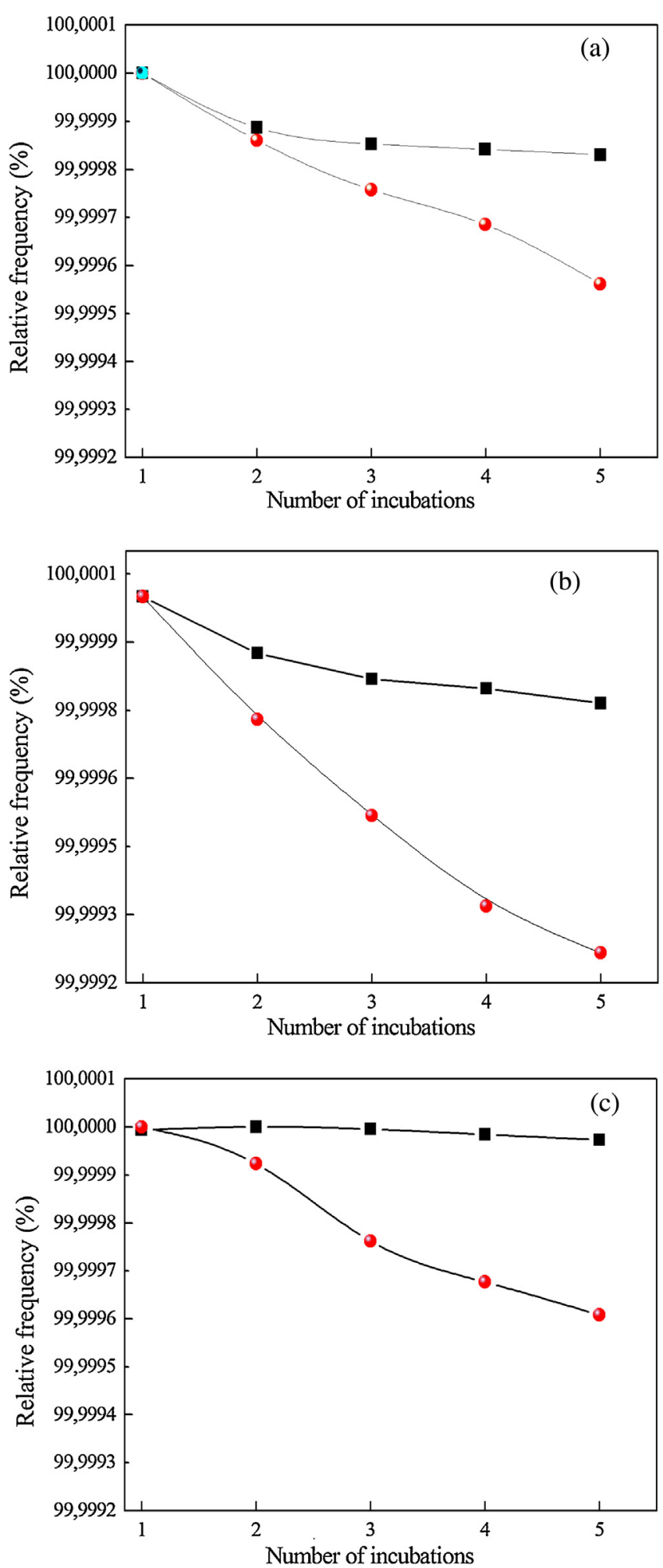

Fig. 6. Blocking of nonspecific binding on response of piezoelectric immunosensor at successive incubations of $(\boldsymbol{\square})$ negative and $(\bullet)$ positive serum samples at dilution of 1:400; comparison of efficiency of blocking solution with $50 \mathrm{mM}$ of glycine (a) $2.5 \%$ albumin (b) and $50 \mathrm{mM}$ of glycine plus $2.5 \%$ albumin (c).

In response to the increasing mass on the crystal surface, there was a decreased frequency of $40.90 \%, 52.27 \%$ and $95.53 \%$ in sera positive for $L$. infantum in relation to negative serum when the system was blocked with a PBS solution containing glycine, BSA or glycine plus BSA, respectively (Table 2).
Table 3

Statistical analysis of immunosensor response according to different dilutions of canine serum positive for anti-L. infantum.

\begin{tabular}{llll}
\hline Serum dilutions & $r$ & Slope value/SE & $p$ \\
\hline $1: 400$ & 0.990 & $-2.0 \mathrm{E}-6 / 1.4 \mathrm{E}-7$ & $\mathbf{7 . 5 E}-\mathbf{4}$ \\
$1: 800$ & 0.998 & $-1.2 \mathrm{E}-6 / 3.8 \mathrm{E}-8$ & $\mathbf{5 . 2 E}-6$ \\
$1: 1600$ & 0.954 & $2.9 \mathrm{E}-7 / 4.6 \mathrm{E}-8$ & $\mathbf{0 . 0 0 8}$ \\
\hline
\end{tabular}

The blocking of nonspecific binding, in which non-target molecules bind to the electrode surface, is an important step in the development of piezoelectric sensors. Washes of the sensor surface prior to reading, in order to remove nonspecifically adsorbed molecules while leaving the target intact, can improve selectivity. A number of studies have reported the blocking activity of nonspecific bonds held by non-target biomolecules (BSA, casein, glycine and gelatin) to avoid false positivity in immunological assays $[51,53]$. Results exhibited in Table 2 indicated that the sensing surface previously exposed to the PBS solution containing glycine and BSA was more effective as a non-specific binding blocking (95.53\%) when compared to use only glycine or BSA, allowing the immunosensor to discriminate canine sera positive and negative for anti-L. infantum antibodies more efficiently. It is likely that the dimensional differences between the two molecules (glycine and BSA) favor the padding of spaces that were not covered by the antigen and were not accessed by a blocking agent when used separately, suggesting an additional blocking action (Fig. 6).

Canine serum contains well over 15 proteins [54] and can therefore be considered a good reference for nonspecific adsorption. Monitoring the uptake of other proteins in canine serum in PBS was used as a test of blocking ability. The blocking solution containing both glycine and BSA was more effective in blocking nonspecific bindings in comparison to each blocking agent employed separately. BSA was chosen due to its standard use as a blocking agent [55]. Small blocking molecules, such as glycine, are preferable to avoid steric hindrance to the further binding of antigens and the detection of antibodies [52].

The specificity of an immunosensor is evaluated with regard to the amount of non-specific bindings that occur on the electrode surface. One way of studying this is by diluting a serum sample to reduce non-specific bindings until the sensor is able to discriminate a positive sample. Table 3 summarizes the data on the responses of the immunosensor incubated with canine serum at different dilutions using Glycine + BSA blocking.

The immunosensor was able to detect anti-L infantum antibodies at different dilutions of the serum sample $(1: 400 ; 1: 800$ and $1: 1600)$. Analysis of the correlation coefficients $(r)$ obtained from the calibration curves with different serum dilution ratios showed that the optimal dilution ratio was found at 1:800, however observing the slope value, the best sensitivity was achieved at 1:400. At this point, it is the best to discriminate canine serum positive for anti-L. infantum antibodies (Table 3). This immunosensor allowed detecting of anti-L infantum antibodies using a lower antigen concentration $(3 \mu \mathrm{g} / \mathrm{mL})$ than that employed with the ELISA method $[56,57]$. The developed immunosensor can be applied to monitor VL through serology, thereby helping control infection in dogs and the consequent transmission of the parasite to humans.

\section{Conclusions}

This immunosensor was able to detect anti- $L$. infantum canine antibodies with a low recombinant antigen concentration ( $3 \mu \mathrm{g} / \mathrm{mL}$ ) without label requirements. The use of AuNP cysteamine modified to immobilize the recombinant antigen was important to increase the electroative area and, consequently increase the sensitivity. This method is simpler, faster and more practical than 
conventional methods. This immunosensor shows as potential for screening of serum samples of endemic areas, consequently helping improve the control of zoonotic visceral leishmaniasis, being a promising tool for application in epidemiological studies and clinical diagnoses of disease.

\section{Acknowledgment}

This work was supported by the Brazilian fostering agency National Council of Scientific and Technological Development (CNPq).

\section{References}

[1] P. Desjeux, Leishmaniasis: current situation and new perspectives, Comp. Immunol. Microbiol. Infect. Dis. 27 (2004) 305-318.

[2] M.C.d.A. Marzochi, S.G. Coutinho, P.C. Sabroza, M.A.d. Souza, P.P.M.d.d. Souza, L. Toledo, F.B. Rangel Filho, Leishmaniose visceral canina no Rio de Janeiro-Brasil, Cadernos de Saú de Pública 1 (1985) 432-446.

[3] C.M. De Souza, E.D. Silva, A.P.D. Ano Bom, R.C. Bastos, H.J. Nascimento, J.G. Da Silva Junior, Evaluation of an ELISA for canine leishmaniasis immunodiagnostic using recombinant proteins, Parasite Immunol. 34 (2012) $1-7$.

[4] S.C. d. Paulan, H.R. Silva, E.A.C.F. d. Lima, E.F. Flores, V.M. Tachibana, C.Z. Kanda, A.C.F. d. Noronha Junior, P.R. Dobre, Spatial distribution of canine Visceral Leishmaniasis in Ilha Solteira, São Paulo, Brazil, Engenharia Agrícola 32 (2012) 765-774.

[5] C.M. de Souza, E.D. Silva, A.P. Ano Bom, R.C. Bastos, H.J. Nascimento, J.G. da Silva Junior, Evaluation of an ELISA for canine leishmaniasis immunodiagnostic using recombinant proteins, Parasite Immunol. 34 (2012) $1-7$.

[6] S.G. Reed, Diagnosis of leishmaniasis, Clin. Dermatol. 14 (1996) 471-478

[7] C. Pirmez, V. da Silva Trajano, M. Paes-Oliveira Neto, A.M. da-Cruz, S.C. Goncalves-da-Costa, M. Catanho, W. Degrave, O. Fernandes, Use of PCR in diagnosis of human american tegumentary leishmaniasis in Rio de Janeiro, Brazil, J. Clin. Microbiol. 37 (1999) 1819-1823.

[8] K.A. Weigle, L.A. Labrada, C. Lozano, C. Santrich, D.C. Barker, PCR-based diagnosis of acute and chronic cutaneous leishmaniasis caused by Leishmanic (Viannia), J. Clin. Microbiol. 40 (2002) 601-606.

[9] J. Disch, M.J. Pedras, M. Orsini, C. Pirmez, M.C. de Oliveira, M. Castro, A. Rabello, Leishmania (Viannia) subgenus kDNA amplification for the diagnosis of mucosal leishmaniasis, Diagn Microbiol. Infect. Dis. 51 (2005) 185-190.

[10] C. Dye, E. Vidor, J. Dereure, Serological diagnosis of leishmaniasis: on detecting infection as well as disease, Epidemiol. Infect. 110 (1993) 647-656.

[11] F. Mancianti, M. Gramiccia, L. Gradoni, S. Pieri, Studies on canine leishmaniasis control. 1. Evolution of infection of different clinical forms of canine leishmaniasis following antimonial treatment, Trans. R. Soc. Trop. Med. Hyg. 82 (1988) 566-567.

[12] C.B. Palatnik-De-Sousa, W.R. Dos Santos, J.C. França-Silva, R.T. Da Costa, A. Barbosa Reis, M. Palatnik, W. Mayrink, O. Genaro, Impact of canine control on the epidemiology of canine and human visceral leishmaniasis in Brazil, Am. J. Trop. Med. Hyg. 65 (2001) 510-517.

[13] R.A. Lira, M.P. Cavalcanti, M. Nakazawa, A.G. Ferreira, E.D. Silva, F.G. Abath, L.C. Alves, W.V. Souza, Y.M. Gomes, Canine visceral leishmaniosis: a comparative analysis of the EIE-leishmaniose-visceral-canina-Bio-Manguinhos and the IFI-leishmaniose-visceral-canina-Bio-Manguinhos kits, Vet. Parasitol. 137 (2006) 11-16

[14] M.M. de Arruda, F.B. Figueiredo, F.A. Cardoso, R.M. Hiamamoto, J.C. Brazuna, M.R. de Oliveira, E.F. Noronha, G.A. Romero, Validity and reliability of enzyme immunoassays using Leishmania major or L. infantum antigens for the diagnosis of canine visceral leishmaniasis in Brazil, PLoS One 8 (2013) e69988.

[15] P. Leonard, S. Hearty, J. Brennan, L. Dunne, J. Quinn, T. Chakraborty, R. O'Kennedy, Advances in biosensors for detection of pathogens in food and water, Enzyme Microb. Technol. 32 (2003) 3-13.

[16] X. Chu, Z.-L. Zhao, G.-L. Shen, R.-Q. Yu, Quartz crystal microbalance immunoassay with dendritic amplification using colloidal gold immunocomplex, Sens. Actuators B: Chem. 114 (2006) 696-704

[17] G. Sauerbrey, Verwendung von Schwingquarzen zur Wägung dünner Schichten und zur Mikrowägung, Z. Physik 155 (1959) 206-222.

[18] R.D. Vaughan, C.K. O'Sullivan, G.G. Guilbault, Development of a quartz crystal microbalance (QCM) immunosensor for the detection of Listeria monocytogenes, Enzyme Microb. Technol. 29 (2001) 635-638.

[19] H. Zeng, H. Wang, F. Chen, H. Xin, G. Wang, L. Xiao, K. Song, D. Wu, Q. He, G Shen, Development of quartz-crystal-microbalance-based immunosensor array for clinical immunophenotyping of acute leukemias, Anal. Biochem. 351 (2006) 69-76.

[20] J. Ramos-Jesus, K.A. Carvalho, R.A. Fonseca, G.G. Oliveira, S.M. Melo, N.M. Alcantara-Neves, R.F. Dutra, A piezoelectric immunosensor for Leishmania chagasi antibodies in canine serum, Anal. Bioanal. Chem. 401 (2011) 917-925.

[21] Z. Shen, H. Yan, F.F. Parl, R.L. Mernaugh, X. Zeng, Recombinant antibody piezoimmunosensors for the detection of cytochrome P450 1B1, Anal. Chem. 79 (2007) 1283-1289.
[22] C. March, J.V. Garcia, A. Sanchez, A. Arnau, Y. Jimenez, P. Garcia, J.J. Manclus, A. Montoya, High-frequency phase shift measurement greatly enhances the sensitivity of QCM immunosensors, Biosens. Bioelectron. 65C (2014) 1-8.

[23] X. Luo, A. Morrin, A.J. Killard, M.R. Smyth, Application of nanoparticles in electrochemical sensors and biosensors, Electroanalysis 18 (2006) 319-326.

[24] A. Sharma, Z. Matharu, G. Sumana, P.R. Solanki, C.G. Kim, B.D. Malhotra, Antibody immobilized cysteamine functionalized-gold nanoparticles for aflatoxin detection, Thin Solid Films 519 (2010) 1213-1218.

[25] R.A. Fonseca, J. Ramos-Jesus, L.T. Kubota, R.F. Dutra, A nanostructured piezoelectric immunosensor for detection of human cardiac troponin $\mathrm{T}$, Sensors (Basel) 11 (2011) 10785-10797.

[26] D.Q. Tang, D.J. Zhang, D.Y. Tang, H. Ai, Amplification of the antigen-antibody interaction from quartz crystal microbalance immunosensors via back-filling immobilization of nanogold on biorecognition surface, J. Immunol. Methods 316 (2006) 144-152.

[27] J.M. Pingarrón, P. Yáñez-Sedeño, A. González-Cortés, Gold nanoparticle-based electrochemical biosensors, Electrochim. Acta 53 (2008) 5848-5866.

[28] P. Yáñez-Sedeño, J.M. Pingarrón, Gold nanoparticle-based electrochemical biosensors, Anal. Bioanal. Chem. 382 (2005) 884-886.

[29] H. Wang, Y. Liu, Y. Yang, T. Deng, G. Shen, R. Yu, A protein A-based orientation-controlled immobilization strategy for antibodies using nanometer-sized gold particles and plasma-polymerized film, Anal. Biochem. 324 (2004) 219-226.

[30] W.Q. Deng, Y. Matsuda, W.A. Goddard 3rd., Bifunctional anchors connecting carbon nanotubes to metal electrodes for improved nanoelectronics, J. Am. Chem. Soc. 129 (2007) 9834-9835.

[31] P. Choi, N.H. Jalani, R. Datta, Thermodynamics and proton transport in nafion: I. membrane swelling, sorption, and ion-exchange equilibrium, J. Electrochem. Soc. 152 (2005) E84-E89.

[32] KUBIAK, \#160 W.W., WANG, \#160 J., Flow injection analysis as a tool for studying polymer modified electrodes, Elsevier Amsterdam PAYS -BAS 1996.

[33] G.G. Oliveira, F.B. Magalhaes, M.C. Teixeira, A.M. Pereira, C.G. Pinheiro, L.R. Santos, M.B. Nascimento, C.N. Bedor, A.L. Albuquerque, W.L. dos-Santos, Y.M. Gomes, E.D. Moreira Jr., M.E. Brito, L.C. Pontes de Carvalho, O.P. de Melo Neto, Characterization of novel Leishmania infantum recombinant proteins encoded by genes from five families with distinct capacities for serodiagnosis of canine and human visceral leishmaniasis, Am. J. Trop. Med. Hyg. 85 (2011) 1025-1034.

[34] R. Badaro, D. Benson, M.C. Eulalio, M. Freire, S. Cunha, E.M. Netto, D. PedralSampaio, C. Madureira, J.M. Burns, R.L. Houghton, J.R. David, S.G. Reed, rK39: a cloned antigen of Leishmania chagasi that predicts active visceral leishmaniasis, J. Infect. Dis. 173 (1996) 758-761.

[35] R. Kumar, K. Pai, K. Pathak, S. Sundar, Enzyme-linked immunosorbent assay for recombinant K39 antigen in diagnosis and prognosis of Indian visceral leishmaniasis, Clin. Diagn. Lab. Immunol. 8 (2001) 1220-1224

[36] D. Du, W. Chen, J. Cai, J. Zhang, H. Tu, A. Zhang, Acetylcholinesterase biosensor based on gold nanoparticles and cysteamine self assembled monolayer for determination of monocrotophos, J. Nanosci. Nanotechnol. 9 (2009) 2368-2373.

[37] T. Sun, L. Wang, N. Li, X. Gan, Label-free electrochemical aptasensor for thrombin detection based on the nafion@graphene as platform, Bioprocess Biosyst. Eng. (2011).

[38] S. Campuzano, R. Gálvez, M. Pedrero, F.J.M. de Villena, J.M. Pingarrón, Preparation, characterization and application of alkanethiol self-assembled monolayers modified with tetrathiafulvalene and glucose oxidase at a gold disk electrode, J. Electroanal. Chem. 526 (2002) 92-100.

[39] J.J. Gooding, D.B. Hibbert, The application of alkanethiol self-assembled monolayers to enzyme electrodes, TrAC Trends Anal. Chem. 18 (1999) 525-533.

[40] R.G. Nuzzo, D.L. Allara, Adsorption of bifunctional organic disulfides on gold surfaces, J. Am. Chem. Soc. 105 (1983) 4481-4483.

[41] H.O. Finklea, L. Liu, M.S. Ravenscroft, S. Punturi, Multiple electron tunneling paths across self-assembled monolayers of alkanethiols with attached Ruthenium(II/III) redox centers, J. Phys. Chem. 100 (1996) 18852-18858

[42] J.V. Staros, R.W. Wright, D.M. Swingle, Enhancement by $\mathrm{N}$-hydroxysulfosuccinimide of water-soluble carbodiimide-mediated coupling reactions, Anal. Biochem. 156 (1986) 220-222.

[43] D. Sehgal, I.K. Vijay, A method for the high efficiency of water-soluble carbodiimide-mediated amidation, Anal. Biochem. 218 (1994) 87-91.

[44] S. Campuzano, M. Pedrero, C. Montemayor, E. Fatás, J.M. Pingarrón, Characterization of alkanethiol-self-assembled monolayers-modified gold electrodes by electrochemical impedance spectroscopy, J. Electroanal. Chem. 586 (2006) 112-121.

[45] D.D. Schlereth, H.-L. Schmidt, Spectroelectrochemical detection of phenothiazine and phenoxazine derivatives covalently bound to self-assembled cystamine monolayers, J. Electroanal. Chem. 380 (1995) $117-125$.

[46] E. Katz, D.D. Schlereth, H.-L. Schmidt, Electrochemical study of pyrroloquinoline quinone covalently immobilized as a monolayer onto a cystamine-modified gold electrode, J. Electroanal. Chem. 367 (1994) 59-70.

[47] X.-Y. Hu, Y. Xiao, H.-Y. Chen, Adsorption characteristics of Fe(CN) 63-/4- on Au colloids as monolayer films on cysteamine-modified gold electrode, J. Electroanal. Chem. 466 (1999) 26-30.

[48] H. Cai, C. Xu, P. He, Y. Fang, Colloid Au-enhanced DNA immobilization for the electrochemical detection of sequence-specific DNA, J. Electroanal. Chem. 510 (2001) 78-85. 
[49] A. Chou, P.K. Eggers, M.N. Paddon-Row, J.J. Gooding, Self-assembled carbon nanotube Electrode arrays: effect of length of the linker between nanotubes and electrode, J. Phys. Chem. C 113 (2009) 3203-3211.

[50] H. Fan, Z. Guo, L. Gao, Y. Zhang, D. Fan, G. Ji, B. Du, Q. Wei, Ultrasensitive electrochemical immunosensor for carbohydrate antigen 72-4 based on dual signal amplification strategy of nanoporous gold and polyaniline-Au asymmetric multicomponent nanoparticles, Biosens. Bioelectron. 64 (2015) $51-56$.

[51] E.J. Moore, M.P. Kreuzer, M. Pravda, G.G. Guilbault, Development of a rapid single-drop analysis biosensor for screening of phenanthrene in water samples, Electroanalysis 16 (2004) 1653-1659.

[52] K. Reimhult, K. Petersson, A. Krozer, QCM-D analysis of the performance of blocking agents on gold and polystyrene surfaces, Langmuir 24 (2008) 8695-8700.

[53] J.-W. Park, S. Kurosawa, H. Aizawa, S.-i. Wakida, S. Yamada, K. Ishihara, Comparison of stabilizing effect of stabilizers for immobilized antibodies on QCM immunosensors, Sensors Actuators B: Chem. 91 (2003) 158-162.
[54] S.G. Calazans, C.R. Daleck, J.J. Fagliari, C.F. Repetti, A.B.d. Nardi, J.H.T. Castro, S.C. Fernandes, J.R.F. César, S.M. Rodigheri, Serum protein concentrations in healthy dogs and dogs with lymphoma by means of sodium dodecil sulphate-polyacrylamide gel electrophoresis (SDS-PAGE), Arquivo Brasileiro de Medicina Veterinária e Zootecnia 61 (2009) 1044-1048.

[55] R.G. Chapman, E. Ostuni, S. Takayama, R.E. Holmlin, L. Yan, G.M. Whitesides, Surveying for surfaces that resist the adsorption of proteins, J. Am. Chem. Soc. $122(2000) 8303-8304$.

[56] G.-H.R. Rajasekariah, L. Cardoso, D.A. Dogcio, S.K. Martin, A.M. Smithyman, A Novel Exo-antigen-based ELISA for the Detection of Canine Leishmaniasis, Am. J. Trop. Med. Hyg. 78 (2008) 616-623.

[57] M. Mohebali, M. Taran, Z. Zarei, Rapid detection of Leishmania infantum infection in dogs: comparative study using an immunochromatographic dipstick rk39 test and direct agglutination, Vet. Parasitol. 121 (2004) 239-245. 